\title{
Spectrum Sharing with Decentralized Occupation Control in Rule Regulated Networks
}

\author{
Alexandr M. Kuzminskiy, Pei Xiao, Rahim Tafazolli
}

\begin{abstract}
Decentralized dynamic spectrum allocation (DSA) that exploits adaptive antenna array interference mitigation diversity at the receiver, is studied for interference-limited environments with high level of frequency reuse. The system consists of base stations (BSs) that can optimize uplink frequency allocation to their user equipments (UEs) to minimize impact of interference on the useful signal, assuming no control over resource allocation of other BSs sharing the same bands. To this end, "good neighbor" (GN) rules allow effective trade-off between the equilibrium and transient decentralized DSA behavior if the performance targets are adequate to the interference scenario. In this paper, we 1) extend the GN rules by including a spectrum occupation control that allows adaptive selection of the performance targets; 2) derive estimates of absorbing state statistics that allow formulation of applicability areas for different DSA algorithms; 3) define a semianalytic absorbing Markov chain model and study convergence probabilities and rates of DSA with occupation control including networks with possible partial breaks of the GN rules. For higher-dimension networks, we develop simplified search GN algorithms with occupation and power control and demonstrate their efficiency by means of simulations.
\end{abstract}

Keywords-Decentralized dynamic spectrum allocation, performance target, occupation control, "selfish" and "good neighbor" rules, absorbing Markov chain.

\section{INTRODUCTION}

To meet the challenge of ever increasing demands for higher capacity and better user experience in wireless communications industry, a number of promising technologies have been suggested in the literature, e.g. in [1] and references therein. One such technology relies on utilizing more frequency resources via, e.g., carrier aggregation, incorporation of unlicensed spectrum, shared-license spectrum, and millimeter wave spectrum [2] - [5]. Another approach is to deploy more network nodes, including both macro BSs and low power nodes such as picos, femtos, small cells, and relays, leading to dense or ultra-dense networks (UDN) [6]. Particularly, coexistence of spectrum sharing UDNs, possibly based on different radio access technologies (RAT) in unlicensed spectrum is considered in [7] as a useful supplement to the conventional $5 \mathrm{G}$ cellular licensed band scenarios.

The main limiting factor for spectrum sharing UDNs is the harsh and generally uncontrollable interference environment, which may compromise user experience leading to a limited applicability of a spectrum sharing technology. Particularly, spectrum sharing is viable in practice: "if shared spectrum can be accessed in a way that gives some assurance of quality" [7]. Clearly, a centralized resource al-

Authors are with the 5G Innovation Center, University of Surrey, UK, (a.kuzminskiy,p.xiao,r.tafazolli)@surrey.ac.uk. Part of this work was presented at PIMRC 2016 [24]. location typical for cellular systems in licensed spectrum is not applicable in this case. Furthermore, even limited coordination among neighboring nodes may be prohibitive, e.g., for multi-RAT coexistence, if this coordination involves a direct data exchange between spectrum sharing nodes even at the medium access control (MAC) level. One way to overcome this difficulty is a rule regulated approach [8] [12], which assumes that spectrum sharing subsystems: 1) can sense the interference environment: 2) have full information and control on their local UEs; 3) agree to follow some predefined rules formulated for the given spectrum band or group of bands. One example of such a rule regulation is carrier sense multiple access collision avoidance (CSMA/CA) protocol and its high efficiency versions [9] [11] for WLAN. A multichannel CSMA/CA extension is addressed in [12] by means of selectively activating or deactivating groups of OFDM subcarriers separated by the guard bands. Generally, CSMA/CA relies on a random backoff that is unable to completely eliminate collisions leading to degradation of the network throughput as more contenders attempt to share the channels.

Our objective in this paper is to study another possibility of rule regulated spectrum sharing based on joint avoidance and antenna array interference mitigation assuming no data exchange between coexisting subsystems. The idea is that if effective antenna array interference mitigation is possible with the available network resources at any subsystem, then UEs can transmit packets sequentially without any additional interference avoidance mechanisms. In turn, this creates a predictable continuous interference environment, where conventional training (pilot) based antenna array interference mitigation techniques can be applied with controllable efficiency.

A general theoretic formulation of the coexisting multiple input multiple output (MIMO) systems is studied in [13] - [17]. The main objective of these studies is to specify conditions that can guarantee convergence of the selfish (SLF) local maximization of the data rate to the unique Nash equilibrium (NE) from which every user is not willing to unilaterally move. Particularly in [13], it is shown that if the channel matrices have full column rank and the multiuser interference is almost negligible, then the NE is unique, without quantifying how small the interference must be. In [14] - [16], the sufficient conditions are found that "explicitly quantify how strong the multiuser interference can be to guarantee both the uniqueness of the equilibrium and the convergence" of asynchronous distributed algorithms for the MIMO game with square non-singular channel matrices. In [17] these conditions are extended to the case of rectangular and possibly rank deficient channel 
matrices. An applicability of these results may be questionable because even if the nodes could know "how strong the multiuser interference can be to guarantee both the uniqueness of the equilibrium and the convergence," it may be difficult or impossible to control the required interference level, which means that network behavior can be arbitrary in the general interference case.

A possibility of arbitrary behavior of selfish (greedy) networks is discussed in the literature, e.g. [19] - [21]. In [20], it is demonstrated that the selfish algorithm, where each player is searching for the maximum data rates, does not necessarily converge to NE. In [21], a selfish channel allocation in wireless networks of coexisting transmitter/receiver pairs was studied by using the generalized spatial congestion games. Special network conditions were found, where existence and convergence to the NE is guaranteed. That is for networks with reciprocal (symmetric) interference scenarios. However, in the general case, the desired behavior cannot be guaranteed. For example, the convergent and non-convergent trials were just counted in different simulation trials in [21] without any suggestions or solutions to the non-convergent situations.

A possibility to improve behavior of selfish DSA in the joint interference avoidance / mitigation scenario is studied in [22] for decentralized DSA in the uplink of interference limited wireless systems consisting of multiple-antenna BSs and single-antenna UEs. These systems do not explicitly cooperate in a centralized fashion and they do not use any direct data exchange between them. Frequency channels (bands) in this case can be formed in an OFDM-based system with guard bands for preventing energy leakage between channels allocated to unsynchronized users similarly to [12] or in spectrally efficient, e.g., filter bank based, multicarrier systems by using frequency selective filters for adjacent channels [25]. Antenna array interference mitigation based DSA at each subsystem should allocate bands to its users, such that the propagation channels from the users to their BSs are as orthogonal as possible to the active interference propagation channels. In [22], this type of allocation is referred to as an antenna array interference mitigation diversity and a rule regulated "good neighbor" (GN) approach to decentralized DSA is proposed that may be treated as useful, despite the fact that convergence with probability one to certain stationary (equilibrium) states cannot be guaranteed. Instead, a trade-off between the equilibrium and transient performance is introduced, which allows a controllable convergence behavior without any explicit communications between spectrum sharing subsystems. The main GN idea is that the controllable performance targets should be reached with minimum changes to the current band allocations ${ }^{1}$. Such minimization of the band switches reduces nonstationary interference in the network leading to faster and more reliable convergence compared to the selfish resource allocation especially for

\footnotetext{
${ }^{1}$ The GN resource allocation could be considered as a general alternative to the selfish network self-organization, e.g. as illustrated in [23] in the downlink beamforming scheduling problem for coexisting multi-RAT networks.
}

systems with a large number of users. Efficiency of the GN DSA is established subject to the performance targets for the given numbers of BS antennas and bands that are adequate to the existing interference scenario. In the realistic spectrum sharing applications, the UE numbers for different BSs with the given number of antennas may be variable leading to unpredictable interference environment for different subsystems. In the case of the performance targets mismatch, the overall network performance may be significantly degraded. For example, if the performance targets are defined under the wrong assumption that the overall number of degrees of freedom allows efficient mitigation of all interference components, then the GN algorithms actually become selfish ones with the corresponding negative consequences. This means that in order to give a practical perspective for the GN algorithms, an adaptive selection of the reliable performance targets needs to be addressed.

Generally, for the requested network occupation (number of activated UEs) per spectrum sharing subsystem and the available resources (number of bands and BS antennas) a potential performance should be estimated leading to the required performance targets. An important observation is that any limited resources cannot serve an arbitrary network occupation. Thus, it is natural to assume availability of other resources, e.g. groups of bands or RATs, and possibility of user reallocation between them leading to a possible difference between the requested and achievable network occupation. Taking into account this general situation, we consider application of the GN DSA to the specific spectrum sharing problem, where the performance targets can be easily established [24]. Particularly, we propose an extension of the GN rules by means of including the spectrum occupation control that allows adaptive selection of the performance targets. The idea is that spectrum sharing subsystems should agree to change the number of their UEs following some fairness restrictions including a possibility to relocate some of the UEs to be served by other resources in the "no room" case for the considered group of available bands. Then, the performance targets can be locally estimated at each sensing interval assuming the noise limited scenario.

To investigate the convergence and equilibrium properties of the proposed spectrum sharing network configuration, we derive absorbing state (AS) statistics and expand the semi-analytic absorbing Markov chain model from [22] to DSA with occupation control. Semi-analytic (analytic for the given network configuration and propagation channel realizations) Markov chain modeling of rule regulated decentralized DSA allows investigation of networks, when convergence to NE (absorbing states in Markov chain terminology) cannot be guaranteed with probability one. Particularly, we study ergodic subchain (ES) statistics, convergence properties, and the data rate for the weakest link in the network for the band allocations corresponding to the absorbing states if they exist for the given scenario realization (equilibrium performance) of the SLF, GN, and mixed $\mathrm{SLF} / \mathrm{GN}$ algorithms. Investigation of the mixed SLF/GN networks illustrates a possibility of significant performance 
degradation because of the rule break effects if some network players are not willing to follow the GN rules. Furthermore, we use the semi-analytic study as a supplement and verification tool for our analytical investigation of the AS statistics. Comparison of the AS and ES statistics allows us to explain behavior and define applicability areas for different algorithms. Particularly, we show that the relatively high and controllable number of ASs establishes statistical constraints on the number of ergodic states in the GN network leading to significantly lower and controllable non-convergence probability compared to the SLF case with its significantly lower and practically uncontrollable number of ASs.

The contribution of this paper compared to [22] is threefold: 1). We extend the GN rules by including a spectrum occupation control that allows adaptive selection of the performance targets. 2). We derive estimates of absorbing state statistics that allow formulation of applicability areas for different DSA algorithms. 3). We define a semi-analytic absorbing Markov chain model and study convergence probabilities and rates of DSA with occupation control including networks with possible partial breaks of the GN rules. For higher-dimension networks, we introduce the simplified GN algorithms with occupation and power control and use simulations to illustrate their efficiency in different scenarios.

The rest of the paper is organized as follows. The system model and problem formulation are presented in Section II. In Section III, the decentralized DSA algorithms with occupation control are introduced. Algorithm analysis is given in Section IV including development of analytical estimates for AS statistics and semi-analytical performance investigation for SLF, GN, and mixed SLF/GN networks. In Section V, the GN algorithms for higher dimension networks with occupation and power control are formulated and studied by means of simulations. Finally, Section VI concludes the paper.

\section{System Model And Problem Formulation}

The considered system consists of $N$ independent subsystems containing base stations $\mathrm{BS}_{n}, n=1, \ldots, N$ and corresponding users $\mathrm{UE}_{n m}, m=1, \ldots, M_{n}$, where $M_{n}$ is the number of activated users per $\mathrm{BS}_{n}$. Single-antenna users transmit data to their BSs equipped with $K$ receive antennas using one of $F$, possibly $F<M_{n}$, available frequency channels. BSs have full information and control of their own users. In particular, they can estimate propagation channels in all the available bands and assign the individual bands and transmit powers to their own users. Base stations do not have any information and control on interference that can be generated by their UEs to other subsystems. Instead, they can asynchronously sense the interference environment.

For simplicity assuming narrowband channels, the signal received by an antenna array of $K$ elements for the $n$th subsystem can be expressed as follows:

$$
\mathbf{x}_{n f}(t)=\sum_{l=1}^{N} \sum_{m=1}^{M_{l}} \delta_{f d_{l m}} q_{l m} \mathbf{h}_{d_{l m} m l n} s_{l m}(t)+\mathbf{z}_{n f}(t),
$$

where $\mathbf{x}_{n f}(t)$ is a $K \times 1$ vector of the signal received at $\mathrm{BS}_{n}$ in the $f$ th band at the $t$ th time instant, $\mathbf{h}_{f m l n}$ is a $K \times 1$ vector of the propagation channel to $\mathrm{BS}_{n}$ in the $f$ th band from the $m$ th user of the $l$ th subsystem, including $l=n$ for the local UEs for $\mathrm{BS}_{n}, s_{n m}(t)$ is the transmitted signal from $\mathrm{UE}_{n m}$ with $\mathrm{E}\left\{\left|s_{n m}(t)\right|^{2}\right\}=1$ and $q_{n m}^{2}$ is the UE transmit power, $Q_{n}=\sum_{m=1}^{M_{n}} q_{n m}^{2}=M_{n}$ is the total power constraint per subsystem, $\mathbf{z}_{n f}(t)$ is a $K \times 1$ vector of AWGN with $\sigma^{2} \mathbf{I}_{K}$ covariance matrix, $d_{n m}$ is the $m$ th element of the $1 \times M_{n}$ vector $\mathbf{d}_{n}$ denoting the frequency band allocation for $\mathrm{UE}_{n m}, \mathrm{E}\{\cdot\}$ is the averaging operator, $(\cdot)^{*}$ is the conjugate transpose operation, $\mathbf{I}_{K}$ is the $K \times K$ unity matrix, and $\delta_{i j}$ is the Kronecker function.

Different global performance metrics can be defined. For example, the total sum data rate:

$$
\gamma=\sum_{n=1}^{N} \sum_{m=1}^{M_{n}} \log _{2}\left[1+\operatorname{SINR}\left(\mathbf{d}_{n}\right)\right]
$$

or the data rate for the weakest link in the network:

$$
\gamma=\min _{n=1, \ldots, N, m=1, \ldots, M_{n}} \log _{2}\left[1+\operatorname{SINR}\left(\mathbf{d}_{n}\right)\right],
$$

where $\operatorname{SINR}\left(\mathbf{d}_{n}\right)=q_{n m}^{2} \mathbf{h}_{d_{n m} m n n}^{*} \mathbf{R}_{d_{n m} n}^{-1} \mathbf{h}_{d_{n m} m n n}$ is the SINR at the output of the optimal spatial filter for $\mathrm{UE}_{n m}$ and

$$
\begin{aligned}
\mathbf{R}_{d_{n m} n} & =\tilde{\mathbf{R}}_{d_{n m} n}+\sum_{p=1, p \neq m}^{M_{n}} \delta_{d_{n m} d_{n p}} q_{n p}^{2} \mathbf{h}_{d_{n p} p n n} \mathbf{h}_{d_{n p} p n n}^{*} \\
\tilde{\mathbf{R}}_{d_{n m} n} & =\sum_{i=1, i \neq n}^{N} \sum_{j=1}^{M_{i}} \delta_{d_{n m} d_{i j}} q_{i j}^{2} \mathbf{h}_{d_{i j} j i n} \mathbf{h}_{d_{i j} j i n}^{*}+\sigma^{2} \mathbf{I}_{K}
\end{aligned}
$$

where $\mathbf{R}_{d_{n m} n}$ is the $K \times K$ total interference covariance matrix at $\mathrm{BS}_{n}$ in the band allocated to $\mathrm{UE}_{n m}$ including the inter-system interference plus noise covariance matrix $\tilde{\mathbf{R}}_{d_{n m} n}$ and the intra-system interference matrix if a number of local UEs occupy the same band as $\mathrm{UE}_{n m}$, which is unavoidable if $F<M_{n}$.

To emphasize DSA fairness, we use the data rate for the weakest link (3) as the performance metric in this paper, but other metrics can be addressed in a similar way.

The total power constraint of $\sum_{m=1}^{M_{n}} q_{n m}^{2}=M_{n}$ is assumed for all spectrum sharing nodes $n=1, \ldots, N$. In the beginning, we assume the constant power $q_{n m}^{2}=1$ for all users in the system. Power control is addressed in Section $\mathrm{V}$.

In the considered network without any centralized processing, the global metrics like (2) and (3) cannot be estimated and maximized. Instead, all subsystems can estimate their local versions and use them for DSA. The basic assumption is that the local information such as $\tilde{\mathbf{R}}_{f n}$ 
and $\mathbf{h}_{f m n n}$ for $f=1, \ldots, F, m=1, \ldots, M_{n}$, can be estimated at $\mathrm{BS}_{n}$ from non-overlapping sensing intervals with the usual assumption that the transmitted data blocks contain the pilot symbols known at the BS. One way to estimate the inter-system interference plus noise covariance matrix is to introduce a mute interval for the local users as a part of the sensing interval. The critical point is that this estimation can be carried out at the sensing BS and it does not require any information and/or cooperation with other BSs. Design of particular sensing/data transmission protocols and analysis of the finite amount of data estimation effects is out of the scope of the current paper. It is worth emphasizing that this basic assumption is relevant for any DSA algorithm based on a local search such as SLF, GN and any their modifications with and without antenna array interference mitigation diversity (in the latest case $\tilde{\mathbf{R}}_{f n}$ and $\mathbf{h}_{f m n n}$ are just replaced with the interference powers and channel gains for local UEs in all bands, e.g. as in [21]). Similarly to [16], [18] and other papers, for convergence analysis, each channel is assumed to change sufficiently slowly to be considered fixed during the whole transmission. Time varying channel effects are illustrated in Section V.C using the IEEE 802.11n channel model [26]. In this paper, we assume that exact estimates of $\tilde{\mathbf{R}}_{f n}$ and $\mathbf{h}_{f m n n}$ are available at $\mathrm{BS}_{n}$. An initial investigation of finite amount of data estimation effects can be found in [27]. This system model is similar to [22] except for some generalization regarding variable $M_{n}$ for different $\mathrm{BS}_{n}$ and arbitrary $F$ and $M_{n}$ relations including $F<M_{n}$. The key symbols used in the paper are summarized in Table 1 .

The problem is to develop and analyze decentralized algorithms for selection of the number of locally served users $M_{n}$ and decision vectors $\mathbf{d}_{n}$ that with high probability achieve reasonably fast convergence to acceptable/controllable equilibrium performance (3) for the given number of bands $F$ and BS receive antennas $K$.

\section{Decentralized DSA With Occupation CONTROL}

\section{A. Basic Algorithms}

At the data intervals, all $\mathrm{UE}_{n m}, n=1, \ldots, N, m=$ $1, \ldots, M_{n}$ transmit data in the bands assigned in $\mathbf{d}_{n} . \mathrm{BS}_{n}$, $n=1, \ldots, N$ receive data with the optimal weight vectors

$$
\mathbf{w}_{n m}=\frac{\mathbf{R}_{d_{n m} n}^{-1} \mathbf{h}_{d_{n m} m n n}}{\mathbf{h}_{d_{n m} m n n}^{*} \mathbf{R}_{d_{n m} n}^{-1} \mathbf{h}_{d_{n m} m n n}}
$$

Table 1. Summary of key symbols / notations

\begin{tabular}{ll}
\hline \hline Symbol & Definition \\
\hline$N$ & Number of subsystems \\
$F$ & Number of frequency bands \\
$K$ & Number antenna array elements at base stations \\
$\mathbf{h}_{f m l n}$ & $K \times 1$ vector of propagation channel \\
$\mathbf{R}_{f n}$ & $K \times K$ covariance matrix of total interference \\
$\tilde{\mathbf{R}}_{f n}$ & $K \times K$ covariance matrix of inter-system interference \\
& plus noise \\
$q_{n m}^{2}$ & User transmit power \\
$Q_{n}$ & Total power per subsystem \\
$\mathbf{d}_{n}$ & $1 \times M_{n}$ vector of band allocations \\
$M_{n}$ & Number of activated users per per subsystem \\
$\tilde{M}_{n}$ & Requested number of users per subsystem \\
$M_{n}^{\text {total }}$ & Total number of activated signals and inter-system \\
$\beta, \delta, \Delta$ & interference sources per subsystem \\
$\gamma_{n}$ & Occupation control parameters \\
$\gamma_{n}^{\text {target }}$ & Minimum data rate at the sensing base station \\
$\alpha, \xi$ & Target threshold \\
$I, \bar{I}$ & Target threshold control parameters \\
$\bar{A}^{L E}$ & Total and effective network dimensions (number of states) \\
\hline \hline
\end{tabular}

At the sensing interval for the basic no-occupation control decentralized DSA, one randomly selected $\mathrm{BS}_{n}$ senses the interference environment, estimates $\tilde{\mathbf{R}}_{f n}, \mathbf{h}_{f m n n}, m=$ $1, \ldots, M_{n}, f=1, \ldots, F$, and updates $\mathbf{d}_{n}$ if needed. Following [22], we consider SLF and GN as the basic DSA algorithms. According to metric (3), at each sensing interval, SLF maximizes the data rate for the weakest UE of the sensing subsystem:

$$
\begin{gathered}
\mathbf{d}_{n}^{S L F}=\arg \max _{d_{n m}=1, \ldots, F} \gamma_{n}\left(\mathbf{d}_{n}\right), \\
\gamma_{n}\left(\mathbf{d}_{n}\right)=\log _{2}\left(1+\min _{m=1, \ldots, M_{n}} \mathbf{h}_{d_{n m} m n n}^{*} \mathbf{R}_{d_{n m} n}^{-1} \mathbf{h}_{d_{n m} m n n}\right) .
\end{gathered}
$$

According to the GN approach, the GN algorithm searches for the local band allocations that achieve some performance target with the minimum changes to the current allocations:

$$
\mathbf{d}_{n}^{G N}= \begin{cases}\mathbf{d}_{n}^{(0)}, & \gamma_{n}\left(\mathbf{d}_{n}^{(0)}\right) \geq \gamma_{n}^{\text {target }} \\ \tilde{\mathbf{d}}_{n}, & \gamma_{n}\left(\mathbf{d}_{n}^{(0)}\right)<\gamma_{n}^{\text {target }}\end{cases}
$$

where $\gamma_{n}\left(\mathbf{d}_{n}\right)$ is the minimum data rate for the $n$th sensing subsystem for band allocation $\mathbf{d}_{n}, \mathbf{d}_{n}^{(0)}$ is the current band allocation, $\gamma_{n}^{\text {target }}$ is the performance target, and

$$
\tilde{\mathbf{d}}_{n}=\arg \min _{d_{n m}=1, \ldots, F} \sum_{m=1}^{M_{n}}\left|\operatorname{sign}\left(d_{n m}-d_{n m}^{(0)}\right)\right|,
$$

$$
\text { subject to } \gamma_{n}\left(\mathbf{d}_{n}\right) \geq \gamma_{n}^{\text {target }},
$$

where the minimization function in (10) is the number of band allocation changes compared to the current allocation, $\operatorname{sign}(a)=\{-1,0,1\}$ is the sign function. If solution (10) does not exist, then $\mathbf{d}_{n}^{G N}=\mathbf{d}_{n}^{S L F}$. One can see that GN and SLF are equivalent for $\gamma_{n}^{\text {target }} \rightarrow \infty$.

\section{B. Target Threshold Selection and Occupation Control}

As mentioned in Section I, selection of the target thresholds $\gamma_{n}^{\text {target }}$ is critical for the GN algorithms. If the total 
number of degrees of freedom available at $\mathrm{BS}_{n}$ exceeds the total number of the local activated signals and the intersystem interference sources $M_{n}^{\text {total }}$ received at $\mathrm{BS}_{n}$

$$
K F>M_{n}^{\text {total }},
$$

then $\gamma_{n}^{\text {target }}$ could be estimated assuming the no intersystem interference scenario:

$$
\gamma_{n}^{\text {target }}=
$$

$\log _{2}\left(1+\alpha \xi \max _{d_{n m} \in[1, F]} \min _{m \in\left[1, M_{n}\right]} \mathbf{h}_{d_{n m} m n n}^{*} \mathbf{R}_{d_{n m} n}^{-1} \mathbf{h}_{d_{n m} m n n}\right)$, for $\tilde{\mathbf{R}}_{d_{n m} n}=\sigma^{2} \mathbf{I}_{K}$ in equation (4) for $\mathbf{R}_{d_{n m} n}$ representing the inter-system free interference scenario, where $\alpha$ is a parameter to balance between the equilibrium and convergence properties, and $\xi$ is the diversity matching coefficient to take into account that a number of degrees of freedom needs to be reserved for interference mitigation and they should be removed from $\gamma_{n}^{\text {target }}$ definition (12) based on the inter-system interference free scenario. The diversity matching coefficient $\xi$ can be defined as a ratio of the actual average diversity gain $K-M_{n}^{\text {total }} / F$ and the interference free average diversity gain $K-M_{n} / F$ leading to:

$$
\xi=\frac{K F-M_{n}^{\text {total }}}{K F-M_{n}} .
$$

To allow target estimation according to (12), condition (11) should be maintained. For that, we introduce the occupation control based on the locally estimated total dimension of the noise subspace of the interference-plus-noise covariance matrices in all bands:

$$
M_{n}^{\text {noise }}=\sum_{f=1}^{F} U_{n f}
$$

where $U_{n f}$ is the dimension of the noise subspace in the $f$ th band defined as the highest index in inequality $\lambda_{n f U_{n f}} \leq$ $\delta \sigma^{2}$, where $\lambda_{n f k}, k=1, \ldots, K$ are increasingly ranked eigenvalues of $\tilde{\mathbf{R}}_{f n}$, and $\delta>1$ is the interference level threshold.

Then, the number of activated UEs at the sensing subsystem can be updated to maintain (11):

$$
M_{n}=\left\{\begin{array}{ll}
M_{n}, & \text { if } M_{n} \leq M_{n}^{\text {noise }}-\beta \text { and } M_{n}=\tilde{M}_{n} \\
M_{n}=M_{n}+\Delta & \text { if } M_{n}<M_{n}^{\text {noise }}-\beta \text { and } M_{n}<\tilde{M}_{n} \\
M_{n}=M_{n}-\Delta & \text { if } M_{n}>M_{n}^{\text {noise }}-\beta
\end{array},\right.
$$

where $\tilde{M}_{n}$ is the requested number of UEs for $\mathrm{BS}_{n}, \beta \geq 0$ is the caution parameter defined to control network occupation representing the targeted overall dimension of the noise subspace, and $\Delta \geq 1$ is the number of UEs that can be relocated at each sensing interval.

Taking into account that $M_{n}^{\text {total }}=M_{n}+K F-M_{n}^{\text {noise }} \leq$ $K F-\beta$ because of $M_{n} \leq M_{n}^{\text {noise }}-\beta$ according to the bottom row in the occupation control algorithm in (15), the diversity matching coefficient (13) can be calculated as

$$
\xi=\frac{M_{n}^{\text {noise }}-M_{n}}{K F-M_{n}} \geq \frac{\beta}{K F-M_{n}} .
$$

A summary of the decentralized GN DSA with occupation control is as follows:

1: Data interval

1.1: $\mathrm{UE}_{n m}, n=1, \ldots N, m=1, \ldots, M_{n}$ transmit data in $\mathbf{d}_{n}^{G N}$ bands.

1.2: $\mathrm{BS}_{n}, n=1, \ldots N$ receive data with weight vectors $\mathbf{w}_{n m}(6)$.

2: Sensing interval

2.1: Randomly select the $n$th sensing subsystem with probability $N^{-1}$.

2.2: Estimate $\tilde{\mathbf{R}}_{d_{n m} n}$ and $\mathbf{h}_{f m n n}$ (not addressed in the paper, assumed known as discussed in Section II).

2.3: Evaluate $M_{n}^{\text {noise }}$ according to (14).

2.4: Update $M_{n}$ according to (15).

2.5: Evaluate $\gamma_{n}^{\text {target }}$ according to (12), (16).

2.6: Evaluate $\gamma_{n}\left(\mathbf{d}_{n}^{(0)}\right)$ according to $(8)$.

2.7: If $\gamma_{n}\left(\mathbf{d}_{n}^{(0)}\right) \geq \gamma_{n}^{\text {target }}$, then $\mathbf{d}_{n}^{G N}=\mathbf{d}_{n}^{(0)}$, else $\mathbf{d}_{n}^{G N}=\tilde{\mathbf{d}}_{n}$ according to (10) calculated over all different allocations $\mathbf{d}_{n}^{(j)}, j=1, \ldots, F^{M_{n}}$.

2.8: Go to 1 .

\section{Analysis of Decentralized DSA with OCCUPATION CONTROL}

\section{A. Markov Chain Modeling}

To formulate a Markov model we assume that all possible $I$ different allocation matrices $\mathbf{D}_{i}=\left[\mathbf{d}_{1 i} ; \ldots ; \mathbf{d}_{N i}\right]$, $i=1, \ldots, I$ form states of the Markov chain. The current state is fully determined by the current band allocations for all activated users in all subsystems. Sensing at one subsystem can either change or not change the band allocations for this subsystem which is equivalent to transition to another state or remaining in the same state. This is a deterministic transition depending on the channel realizations, the algorithm and its parameters. The random nature of Markov chain is then associated with the randomness of the sequence of band allocation updates among all BSs in the network that in turn, specifies transition probabilities between the states. To take into account the occupation control additionally to band allocations, we introduce one more selection option for each element of the decision vector that reflects activation of the corresponding UE $d_{n m} \in\{0,1, \ldots, F\}$, where " 0 " corresponds to the nonactivated situation. Also, for simplicity we assume $M_{n} \geq 1$, $\tilde{M}_{n}=\tilde{M}$ and place all non-activated UEs at the end of the UE list. For example, vector $\mathbf{d}_{n i}=\left[f_{1 i}, f_{2 i}, 0,0\right]$ indicates that $\tilde{M}=4, M_{n}=2$, and bands $f_{1 i}, f_{2 i}$ are used for activated $\mathrm{UE}_{n 1}$ and $\mathrm{UE}_{n 2}$ correspondingly for the $i$ th Markov chain state. Also, we assume that UEs activation/deactivation just follows the fixed list of UEs for each subsystem without any optimization according to some selection criteria. This simplification reduces the total dimension of the model from $I=(F+1)^{\tilde{M} N}$ in the general case with occupation control to the significantly lower number of $I=\left(\sum_{m=0}^{\tilde{M}-1} F^{\tilde{M}-m}\right)^{N}$.

For a given state $\mathbf{D}_{i}$, sensing at the $n$th subsystem transfers the system to state $\mathbf{D}_{j_{n}}$, where $j_{n} \in[1, I]$, including 
$j_{n}=i$. Sensing at $\mathrm{BS}_{n}$ means that for the given channel realizations, matrices $\tilde{\mathbf{R}}_{f n}, f=1, \ldots, F$ are calculated as in (5), $M_{n}$ is adjusted according to (14), (15), and $\mathbf{d}_{n}$ is updated for the given algorithm as in (7)-(10) leading to $\mathbf{D}_{j_{n}}$. Repeating this procedure for $n=1, \ldots, N$, a set of $\mathbf{D}_{j_{n}}$ can be found for each initial $\mathbf{D}_{i}$, where not all $j_{n}$ may be different.

Assuming that, at each sensing interval, one randomly selected subsystem is sensed with the uniform probability of $N^{-1}$, the nonzero elements of the $I \times I$ transition probability matrix $\mathbf{P}=\left\{p_{i j}\right\}$ can be defined as $p_{i j}=g_{j} / N$, $i=1, \ldots, I$, where $1 \leq g_{j} \leq N$ is the number of sensing outcomes at $\mathrm{BS}_{n}, n=1, \ldots, N$, leading to $\mathbf{D}_{j_{n}}=\mathbf{D}_{j}$.

The transition probability matrix $\mathbf{P}=\left\{p_{i j}\right\}$ is a sparse stochastic matrix such that $\sum_{j=1}^{I} p_{i j}=1$ for $i=1, \ldots, I$, which completely defines the Markov model of the considered spectrum sharing network with the decentarlized DSA and occupation control. The difference of this model compared to the one in [22] with no occupation control is the extended dimension of the decision vectors and the corresponding extension of the total number of Markov model states, which is $I=F^{\tilde{M} N}$ in the case of no occupation control. Now, each absorbing state may have different numbers of the activated UEs for different BSs corresponding to the target performance threshold for the particular interference scenario observed at the sensing intervals.

The state classification algorithm [28] can be used similarly to [22] to replace the ergodic subchains if they exist with the corresponding virtual absorbing states. Then, the absorption probabilities matrix $\mathbf{E}$ from each transient state to each absorbing state and collapsed ergodic subchains, if they exist, and the average number of sensing intervals before absorption $\mathbf{t}$ can be calculated as follows [29]: $\mathbf{E}=\mathbf{C B}, \mathbf{t}=\mathbf{C 1}$, where $\mathbf{C}=\left(\mathbf{I}_{I_{T}}-\mathbf{A}\right)^{-1}$ is the fundamental matrix of the canonical form $\overline{\mathbf{P}}_{A}$ of the absorbing transition probability matrix $\mathbf{P}_{A}$ found after replacement of all ergodic subchains in matrix $\mathbf{P}$, if they exist, with the corresponding absorbing states

$$
\overline{\mathbf{P}}_{A}=\left[\begin{array}{cc}
\mathbf{A} & \mathbf{B} \\
\mathbf{0} & \mathbf{I}_{I_{A}}
\end{array}\right],
$$

1 is the vector of all ones, $I_{T}$ and $I_{A}$ are the numbers of the transient and absorbing states, including the collapsed ergodic subchains in $\mathbf{P}_{A}$ if they exist, and $I_{T}+I_{A} \leq I$. A probability of absorption by ergodic subchains (nonconvergence probability) can be find as

$$
p_{E S}=\sum_{i=1}^{I_{E S}} \mathbf{e}_{E S}^{T}(i) \mathbf{1},
$$

where $I_{E S}$ is the number of ESs in the network, $\mathbf{e}_{E S}(i)$ is the column of matrix $\mathbf{E}$ corresponding to the $i$ th collapsed $\mathrm{ES}$, and $(\cdot)^{T}$ is the matrix transposition operation.

\section{B. Network Scenario for Analytical and Semi-Analytical Investigation}

Propagation channels $\mathbf{h}_{f m l n}$ for all users, bands and subsystems in the network are assumed to be independent ran- dom Gaussian $K \times 1$ vectors

$$
\mathbf{h} \sim \mathcal{C N}\left(0, \sigma_{h}^{2}, \mathbf{I}_{K}\right),
$$

where $\sigma_{h}^{2}=1$. Equation (19) implies a fast Rayleigh fading no pathloss scenario, which can be considered as an example of ultimate UDN interference limited scenario with no difference between the desired and interference signals in terms of power leading to $M_{n}^{\text {total }}=M^{\text {total }}=\sum_{n=1}^{N} M_{n}$. In [30], the same model is called a "strong cross-channels" one and used for comparison of distributed MIMO beamforming algorithms by means of simulations. In the simulation study in Section V, we expand this model assuming that $\sigma_{h}^{2}$ denotes a random variable according to some pathloss and shadowing models for particular network geometries.

This model allows an analytical description of the SINR distribution. Particularly, if the number of antennas exceeds the total number of received signals per band $K \geq$ $M_{0}$, then

$$
\begin{gathered}
\mathbf{R}=\sigma_{i n t}^{2} \mathbf{H}_{M_{0}-1} \mathbf{H}_{M_{0}-1}^{*}+\sigma^{2} \mathbf{I}_{K}, \\
\nu=\mu_{s} \mathbf{h}_{0}^{*}\left(\mathbf{I}_{K}+\mu_{i n t} \mathbf{H}_{M_{0}-1} \mathbf{H}_{M_{0}-1}^{*}\right)^{-1} \mathbf{h}_{0},
\end{gathered}
$$

where $\nu$ denotes the normalized SINR, $\mathbf{R}$ is the interference plus noise covariance matrix, $\mathbf{h}_{0}$ is the propagation channel of the desired signal, $\mathbf{H}_{M_{0}-1}=\left[\mathbf{h}_{1}, \ldots, \mathbf{h}_{M_{0}-1}\right]$ is the matrix of $M_{0}-1$ propagation channels of the co-channel interference, $\left[\mathbf{h}_{0}, \mathbf{h}_{1}, \ldots, \mathbf{h}_{M_{0}-1}\right] \sim \mathcal{C} \mathcal{N}_{K, M_{0}}\left(0, \mathbf{I}_{K}\right), \mu_{s}=\sigma_{s}^{2} / \sigma^{2}$ and $\mu_{i n t}=\sigma_{\text {int }}^{2} / \sigma^{2}$ are the signal to noise (SNR) and interference to noise (INR) ratios $\left(\mu_{s}=\mu_{i n t}=\sigma^{-2}\right.$ for $\left.\sigma_{h}^{2}=1\right)$. According to the matrix inversion lemma [31], for $\mu_{\text {int }} \gg 1$ , equation (21) can be expressed as

$$
\begin{gathered}
\nu=\mu_{s} \mathbf{h}_{0}^{*} \times \\
{\left[\mathbf{I}_{K}-\mathbf{H}_{M_{0}-1}\left(\mathbf{H}_{M_{0}-1}^{*} \mathbf{H}_{M_{0}-1}+\mu_{i n t}^{-1} \mathbf{I}_{M_{0}-1}\right)^{-1} \mathbf{H}_{M_{0}-1}^{*}\right] \mathbf{h}_{0} \approx} \\
\mu_{s} \mathbf{h}_{0}^{*}\left[\mathbf{I}_{K}-\mathbf{H}_{M_{0}-1}\left(\mathbf{H}_{M_{0}-1}^{*} \mathbf{H}_{M_{0}-1}\right)^{-1} \mathbf{H}_{M_{0}-1}^{*}\right] \mathbf{h}_{0} .
\end{gathered}
$$

The normalized $\operatorname{SINR} \nu$ is distributed according to the $\chi^{2}$ law with $2\left(K-M_{0}+1\right)$ degrees of freedom [32]:

$$
\omega(y)=\Gamma\left(K-M_{0}+1\right)^{-1} y^{K-M_{0}} e^{-y},
$$

where $\Gamma(a)$ is the gamma function [33].

Then, the SINR cumulative distribution function (CDF) can be expressed using the table integral from [33] as

$$
\begin{aligned}
& \operatorname{Prob}(\operatorname{SINR}>x)=P\left(\frac{x}{\mu_{s}}, M_{0}\right)= \\
& \int_{\frac{x}{\mu_{s}}}^{\infty} \omega(y) d y=e^{-\frac{x}{\mu_{s}}} \sum_{j=0}^{K-M_{0}} \frac{1}{j !}\left(\frac{x}{\mu_{s}}\right)^{j} .
\end{aligned}
$$

A similar scenario is used in [22] for potential performance analysis in DSA with antenna array interference mitigation diversity. In this paper, the presented network scenario and CDF (24) are to be used for analysis of absorbing state statistics and semi-analytic performance investigation for the developed decentralized DSA algorithms 
with occupation control. The general scenario and GN parameters used for semi-analytic investigation are: $N=3$, $F=2, \tilde{M}=4, \sigma^{2}=10^{-2}, \delta=2, \Delta=1$ and $I=27000$. The particular values of the number of antennas $K$ and the occupation control parameter $\beta$ leading to different values of $M^{\text {total }}$ as well as values of the GN trade-off control parameter $\alpha$ are indicated separately for each case.

\section{Analysis of Absorbing State Statistics}

Comparison of absorbing state and ergodic subchain statistics allows to understand efficiency and find applicability areas for different algorithms. We study AS and ES statistics in the network scenario from Section IV.B and verify our findings by means of semi-analytic Markov chain performance evaluation in Section IV.E and simulations in Section V.

We assume the occupation control with some caution parameter $\beta>0$ in (14), (15), which means that condition (11) is satisfied for

$$
M^{\text {total }}=K F-\beta
$$

For AS statistics analysis we define the following sets of different distributions of $M^{\text {total }}$ UEs in the network between subsystems and bands:

- Set of all possible UE activation patterns:

$$
\begin{gathered}
\mathcal{M}=\left\{\mathcal{M}_{i}\right\}, \\
\mathcal{M}_{i}=\left[M_{i 1}, \ldots, M_{i N}\right], i=1, \ldots, B\left(M^{\text {total }}, N, 1, \tilde{M}\right),
\end{gathered}
$$

where $1 \leq M_{i n} \leq \tilde{M}$ is the number of activated UEs at the $n$th subsystem for the $i$ th activation pattern, and $B\left(M^{\text {total }}, N, 1, \tilde{M}\right)$ is the number of activation patterns for a network of $N$ subsystems with $M^{\text {total }}$ activated UEs with minimum 1 and maximum $\tilde{M}$ UEs activated per subsystem. - Set of all possible band allocation patterns for each activation pattern:

$$
\overline{\mathcal{M}}=\left\{\overline{\mathcal{M}}_{j}\right\}
$$

$\overline{\mathcal{M}}_{j}=\left[M_{j 1}, \ldots, M_{j F}\right], j=1, \ldots, B\left(M^{\text {total }}, F, 0, M^{\text {total }}\right)$, where $0 \leq M_{j f} \leq M^{\text {total }}$ is the number of UEs in the whole network allocated to the $f$ th band for the $j$ th allocation pattern, and $B\left(M^{\text {total }}, F, 0, M^{\text {total }}\right)$ is the number of band allocation patterns for a network of $F$ bands with $M^{\text {total }}$ activated UEs with minimum 0 and maximum $M^{\text {total }} \mathrm{UEs}$ activated per band.

Both $B\left(M^{\text {total }}, N, 1, \tilde{M}\right)$ and $B\left(M^{\text {total }}, F, 0, M^{\text {total }}\right)$ can be found as the number of restricted compositions of $a$ in $b$ groups with minimum $c_{1}$ and maximum $c_{2}$ components in each group with the recurrent and closed form expressions available, e.g. in [34]:

$$
B\left(a, b, c_{1}, c_{2}\right)=\sum_{i=c_{1}}^{c_{2}} B\left(a-i, b-1, c_{1}, c_{2}\right)
$$

with the initialization $B\left(d, 1, c_{1}, c_{2}\right)=\left\{\begin{array}{cc}1 & c_{1} \leq d \leq c_{2} \\ 0 & d>c_{2}, d<c_{1}\end{array}\right.$

We find the number of states $S_{\overline{\mathcal{M}}_{j}}$ with the $j$ th band allocation pattern $\overline{\mathcal{M}}_{j}$ in (27) taking into account that for each activation pattern $\mathcal{M}_{i}$ in (26), $M_{j 1}$ out of $M^{\text {total }} \mathrm{UEs}$ can be allocated to one band with $C_{M^{\text {total }}}^{M_{\text {tal }}}$ combinations, $M_{j 2}$ out of $M^{\text {total }}-M_{j 1}$ UEs can be allocated to another band with $C_{M^{\text {total }}-M_{j 1}}^{M_{21}}$ combinations, and so on until the $F$ th band with $C_{M^{\text {total }}-\sum_{f=1}^{M_{j F}} M_{j f}}$ combinations. Then, $S_{\overline{\mathcal{M}}_{j}}$ can be expressed as

$$
S_{\overline{\mathcal{M}}_{j}}=B\left(M^{\text {total }}, N, 1, \tilde{M}\right) C_{M^{\text {total }}}^{M_{j 1}} \prod_{f=2}^{F} C_{M^{\text {total }}-\sum_{\phi=1}^{f-1} M_{j \phi}}^{M_{j f}},
$$

where $C_{n}^{k}=\frac{n !}{k !(n-k) !}$ is the number of combinations of $k$ from $n$.

The uniform band allocation $M_{0}=\left\lceil M^{\text {total }} / F\right\rceil$ may be of special interest. If the network configuration leads to $M_{0}=M^{\text {total }} / F$, then the number of states with the uniform band allocation can be found as the special case of (29):

$$
S_{\text {uniform }}=B\left(M^{\text {total }}, N, 1, \tilde{M}\right) \prod_{f=0}^{F-1} C_{M_{0}(F-f)}^{M_{0}} .
$$

From (29), it follows that the total number of states with $M^{\text {total }}$ activated UEs in the network is

$$
\bar{I}=\sum_{j=1}^{B\left(M^{\text {total }}, F, 0, M^{\text {total }}\right)} S_{\overline{\mathcal{M}}_{j}}=B\left(M^{\text {total }}, N, 1, \tilde{M}\right) F^{M^{\text {total }}} .
$$

We will refer to $\bar{I}$ as the effective network dimension, which is generally lower than the total number of states $I=\left(\sum_{m=0}^{\tilde{M}-1} F^{\tilde{M}-m}\right)^{N}$ defined in Section IV.A. One can see that activation of all UEs without any occupation control leads to $B(\tilde{M} N, N, \tilde{M}, \tilde{M})=1$ and $\bar{I}=I=F^{\tilde{M} N}$.

Generally, different band allocations cannot be treated as independent since some subsets of them may be repeated. In the following statistical analysis, we ignore this dependency and treat different states as independent leading to approximate nature of our statistical estimates to be verified by simulations.

\section{C.1 SLF}

A SLF AS corresponds to the search outcome for some state, where sensing at each subsystem do not change the number of active users and their band allocations leading to probability one for this state in the transition probability matrix. Clearly, the outcome of local search at some state depends on the number of antennas. For low number of antennas, the probability of selection of the allocations with the lowest number of activated signals is high leading to transitions mainly between states with uniform band occupation. For higher number of antennas, this constraint is relaxed because probability of allocation selection with the lowest number of activated UEs is quickly decreasing with growing number of antennas. Indeed, taking into account that SINR is distributed according to the $\chi^{2}$ law (23) and a ratio of two independent $\chi^{2}$ distributed random values 
scaled with their corresponding numbers of degrees of freedom is distributed according to the $\mathcal{F}$-distribution [35], a probability to find the lowest SINR in allocation with $M_{0}$ UEs per band compared to allocation with $M_{0}+m$ UEs for $1 \leq m \leq K-M_{0}$ can be expressed as

$$
\operatorname{Prob}\left[\nu\left(K, M_{0}\right)>\nu\left(K, M_{0}+m\right)\right]=1-z_{0},
$$

where $z_{0}$ is the root of equation

$$
\mathcal{F}\left[z, 2\left(K-M_{0}+1\right), 2\left(K-M_{0}-m+1\right)\right]=\frac{K-M_{0}-m+1}{K-M_{0}+1},
$$

and $\mathcal{F}(z, a, b)$ is the CDF for $\mathcal{F}$-distribution. The probability function in (32) is plotted in Fig. 1 for $m=1,2,3$ depending on $K-M_{0}$. One can see that although a very higher number of antennas for the given $M_{0}$ is needed to approach probability of 0.5 , but it is quickly decreasing for low $K-M_{0}$ leading to a practically arbitrary selection options even for relatively low $K>M_{0}$.

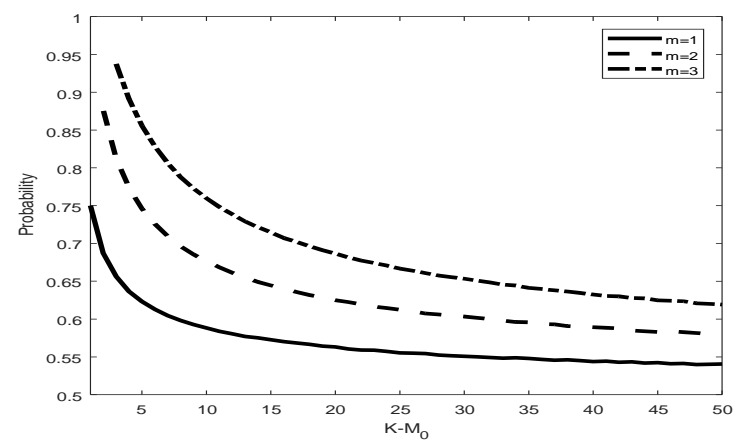

Fig. 1. Probability of selection allocation with the lowest number of activated UEs

This situation allows us to consider an estimate of the number of SLF ASs corresponding to the extreme case when the antenna array size tends to infinity leading to the uniform probability of selection of any state out of all available options of $\bar{I}$ for the given UE activation pattern $p_{S L F}=F^{-M^{\text {total }}}$. We refer to the corresponding statistics of the number of SLF ASs as the lower estimate (LE) because for a finite number of antennas, a number of the actual selection options should be lower leading to higher $p_{S L F}$ compared to the indicated extreme case. Then, the probability to obtain $A_{S L F}^{L E}$ ASs in the network is the probability to have $A_{S L F}^{L E}$ events with probability $p_{S L F}$ in $\bar{I}$ trials, which is

$$
p\left(A_{S L F}^{L E}\right)=C_{\bar{I}}^{A_{S L F}^{L E}} p_{S L F}\left(1-p_{S L F}\right)^{\bar{I}-A_{S L F}^{L E}}
$$

according to Bernoulli formula with the average number of ASs of

$$
\bar{A}_{S L F}^{L E}=p_{S L F} \bar{I}=B\left(M^{\text {total }}, N, 1, \tilde{M}\right) .
$$

Estimates (34) and the actual AS CDFs in 100 channel realizations for different values of $K$ and $\beta$ leading to two groups of scenarios from Section IV.B with $M^{\text {total }}=[8,12]$ are plotted in Fig. 2. The estimates for the average number of ASs according to $(35)$ are: $B(8,3,1,4)=12$ and
$B(12,3,1,4)=1$. As expected, the higher number of antenna results are closer to the lower estimate in both groups. Actually, the estimate is reasonably close to the simulation results for $\beta>2$, although it is derived assuming a very high number of antennas, which confirms our expectation based on (32) statistics.

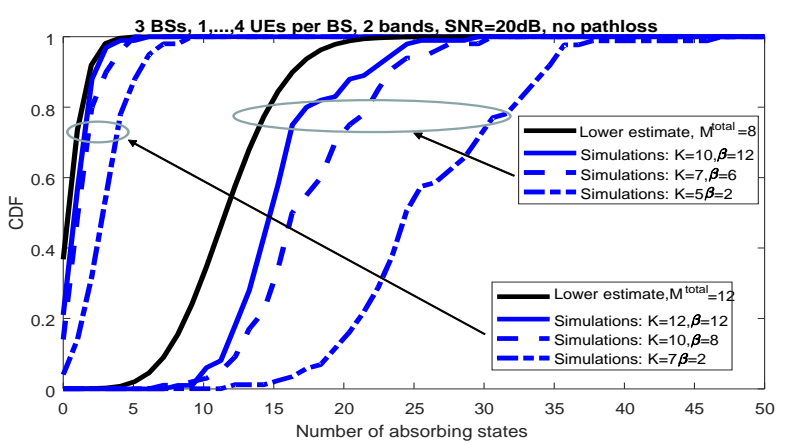

Fig. 2. Absorbing state statistics for SLF

The main observation from the AS SLF statistics analysis is that the relative average number of ASs may be very low for realistic network configurations

$$
\frac{\bar{A}_{S L F}^{L E}}{\bar{I}}=F^{-M^{\text {total }}} \ll 1,
$$

which is the main reason of the undesirable SLF convergence behavior. Next, we will show how this situation is changed in the GN DSA algorithm.

\section{C.2 GN}

GN absorbing states correspond to the states where SINR exceeds the target thresholds for the weakest UEs in all subsystems. Although according to (12) $\gamma_{n}^{\text {target }}$ is calculated locally at each subsystem, to get tractable expressions for GN AS statistics we assume the same target threshold for all subsystems $\gamma_{n}^{\text {target }}=\gamma^{\text {target }}$, which is adequate to the no-pathloss scenario in Section IV.B. Still assuming the independence assumption and taking into account that condition of SINR being higher than some threshold for the weakest UE is equivalent to that it should be higher for all the considered UEs, the average number of the GN absorbing states can be expressed as:

$$
\bar{A}_{G N}=\sum_{j=1}^{B\left(M^{\text {total }}, F, 0, M^{\text {total }}\right)} p_{\overline{\mathcal{M}}_{j}} S_{\overline{\mathcal{M}}_{j}},
$$

where $\overline{\mathcal{M}}_{j}$ and $S_{\overline{\mathcal{M}}_{j}}$ are defined in (27) and (29) correspondingly, and $p_{\overline{\mathcal{M}}_{j}}$ is the probability that SINR exceeds the target threshold for all UEs in the $j$ th allocation

$$
p_{\overline{\mathcal{M}}_{j}}=\prod_{f=1}^{F} P\left(\frac{\bar{\gamma}^{\text {target }}}{\mu_{s}}, M_{j f}\right)^{M_{j f}}
$$

where $P\left(\frac{\bar{\gamma}^{\text {target }}}{\mu_{s}}, M_{j f}\right)$ is the SINR CDF assuming the same threshold for all subsystems $\bar{\gamma}^{\text {target }}=2^{\gamma^{\text {target }}}-1$. 
It is clear that for a reasonably high target selection the main contribution in (37) bring allocations with $M_{j f} \leq K$ for $f=1, \ldots, F$. Then, statics (24) can be used in (38). Furthermore, a uniform allocation component in the sum (37) with $M_{0}$ UEs per band can be used as a lower estimate for the average number of the GN absorbing states:

$$
\begin{gathered}
\bar{A}_{G N}^{L E}=B\left(M^{\text {total }}, N, 1, \tilde{M}\right) \times \\
{\left[e^{-\frac{\bar{\gamma}^{\text {target }}}{\mu_{s}}} \sum_{j=0}^{K-M_{0}} \frac{1}{j !}\left(\frac{\bar{\gamma}^{\text {target }}}{\mu_{s}}\right)^{j}\right]^{M_{0} F} \times} \\
\prod_{f=0}^{F-1} C_{M_{0}(F-f)}^{M_{0}}<\bar{A}_{G N}
\end{gathered}
$$

with CDF (24) in (38) and the number of uniform allocations $S_{\text {uniform }}$ according to (30).

The efficiency of estimate (37) and applicability of the lower estimate (39) in 100 scenario realizations for $K=8$, $\beta=8, M^{\text {total }}=8$ with different fixed target thresholds is illustrated in Fig. 3. In this case, $M_{0}=4, B(8,3,1,4)=12$ and $B(8,2,0,8)=9$ with $\overline{\mathcal{M}}_{j}, j=1, \ldots, 9$ : [4;4], [5;3], $[3 ; 5],[6 ; 2],[2 ; 6],[7 ; 1],[1 ; 7],[8 ; 0],[0 ; 8]$ UEs activated in each band with the corresponding number of states for the given allocations $S_{\overline{\mathcal{M}}_{j}}=12 \cdot[70,56,56,28,28,8,8,1,1]$ and $\bar{I}=\sum_{j=1}^{9} S_{\overline{\mathcal{M}}_{j}}=3072$. In Fig. 3, one can observe that: $1)$. The analytical average number of the GN absorbing states (37) and lower estimate (39) reliably represent the average value of the estimated distributions over all and the uniform allocation states correspondingly for different target thresholds. 2). The analytical and simulation results show that the number of the GN absorbing states can be a significant fraction of the effective network dimension and it can be effectively controlled by selection of the target threshold.

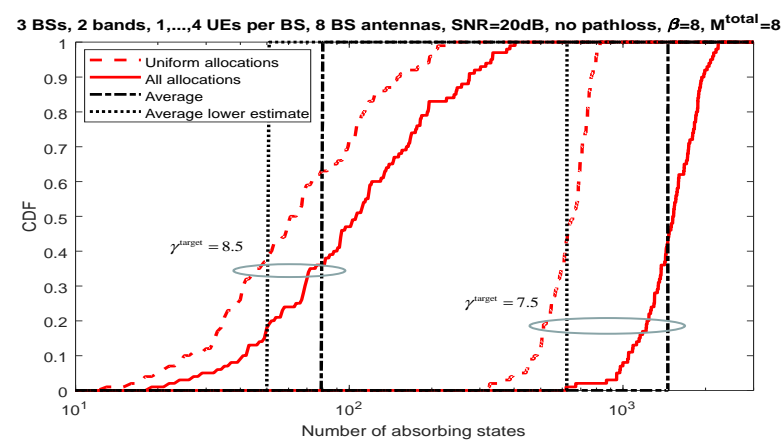

Fig. 3. Absorbing state statistics for GN

It is important to emphasize that the latest observation is relevant for any network dimension as it follows from (37) taking into account that $S_{\overline{\mathcal{M}}_{j}}$ grows with the network dimension according to (29). This makes GN to be profoundly different compared to SLF, which may have a low number of absorbing states for any network dimension at least for relatively high number of antennas as shown in Section IV.C.1.

\section{Ergodic Subchains: Semi-Analytic Study}

The specified difference in the AS statistics for SLF and GN should directly affect the properties of their ES . Indeed, the probability of appearance of ES of $L$ states $p_{E S}(L)$ can be bounded with the probability of no ASs among $L$ states:

$$
p_{E S}(L)<p_{n o A S}(L),
$$

where $p_{n o A S}(L)$ is the probability that exactly zero ASs appear in $L$ randomly selected states. Assuming the same simplifications as in Section IV.C, we have $p_{n o A S}(L) \approx(1-$ $\left.p_{A S}\right)^{L}$ according to Bernoulli formula. For SLF with relatively high number of antennas $p_{A S}=p_{S L F}=F^{-M^{\text {total }}}$ leading to $p_{E S}(L)<\left(1-F^{-M^{\text {total }}}\right)^{L} \approx 1-L F^{-M^{\text {total }}} \approx 1$ even if $L$ is as high as a few percent of $F^{-M^{\text {total }}}$. Although, for the given network configuration, selection of higher values of the occupation control parameter $\beta$ leading to lower values of $M^{\text {total }}$ should reduce probability of longer ESs, appearance of SLF ASs practically should not restrict a possibility of relatively long SLF ESs.

The situation becomes critically different in the GN case, where the number of ASs grows with the network dimension depending on the target threshold, which follows from estimates (37) and (39). For example, if for the given network dimension, we select the target threshold leading to $p_{A S}=0.1$, then similarly to the SLF case above, we can find $p_{E S}(10)<0.35$ and $p_{E S}(30)<0.04$ independently on the network dimension. Actually, this situation illustrates statistical restrictions on the absolute length of ESs leading to high probability of short GN subchains for any network dimensions. Then, even for scenario realizations with ESs one could expect that probability of non-convergence should be decreasing with growing effective network dimensions.

To verify these expectations, we use the semi-analytical tool summarized in Section IV.A and apply it in the scenario in Section IV.B. Clearly, only relatively low dimension networks can be studied this way. Our objective in this section is thus to study performance trends that could be observed and verified for higher dimension networks by simulations rather than investigation of the particular performance in the considered scenarios.

First, we consider 100 realizations of scenario in Section IV.B for $K=7$ and different occupation control parameters of $\beta=[6,2]$ leading to $M^{\text {total }}=[8,12]$, and $\bar{I}=[3072,4096]$. The ES parameters (number of subchains per scenario realization and their lengths) for SLF and GN with the target threshold defined in (12), (16) for $\alpha=0.75$ are shown in Fig. 4. The results in Fig. 4 show that: 1). On average, a low number of ESs is observed for both SLF and GN. 2). Wide range of ESs lengths is observed for SLF up to a few percent of the effective network dimension for both $\beta$ values, although shorter on average ESs are found for $M^{\text {total }}=8$ compared to $M^{\text {total }}=12$ case as expected. 3). Only very short ESs of 4-6 elements are found for GN in both cases as expected.

Fig. 5 presents the ES properties in 100 realizations of 

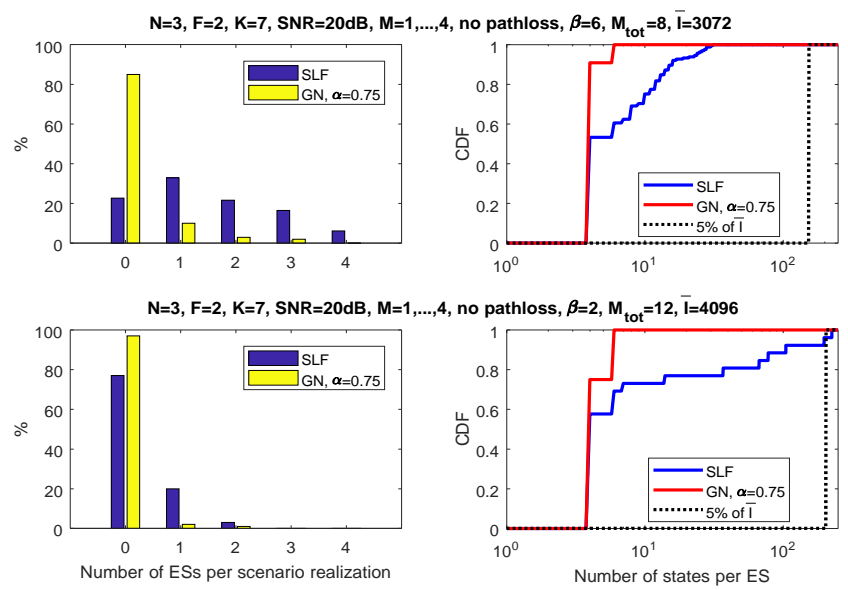

Fig. 4. Ergodic subchain parameters for different occupation control parameters

scenarios with significantly different effective dimensions for $\beta=3$ with different $K=[6,9]$ and $\tilde{M}=[3,5]$ leading to different $M^{\text {total }}=[9,15]$ and $\bar{I}=[512,32768]$. Again, one can see that relatively long SLF ESs and very short GN ESs appear in both cases. The non-convergence probabilities in scenario realizations with ESs calculated according to (18) are plotted in Fig. 6 illustrating our expectation of lower GN non-convergence probabilities for higher dimension networks in scenario realizations with ESs.
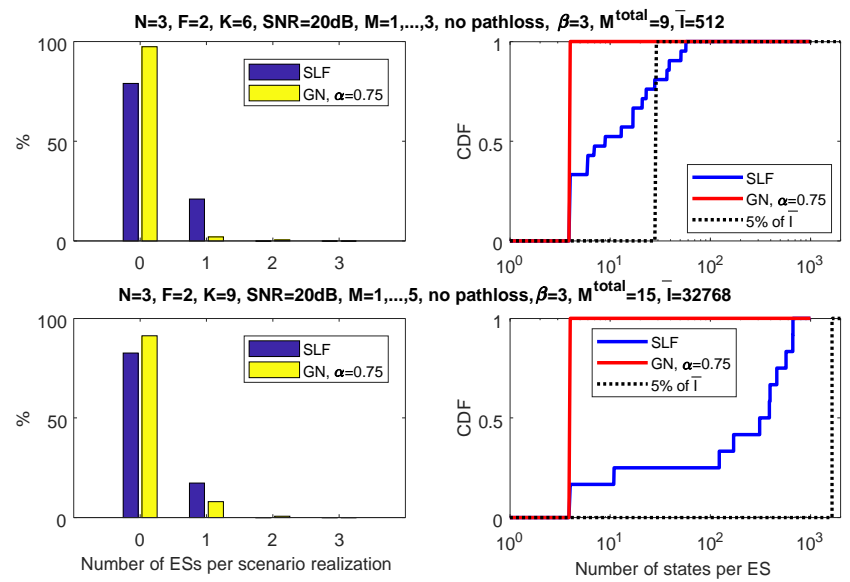

Fig. 5. Ergodic subchain parameters for different effective network dimensions

\section{E. Semi-Analytic Performance Evaluation}

In the beginning, we study the GN behavior with the target threshold defined in (12), (16) without occupation control when condition (11) is satisfied or may be violated. We assume $M_{n}=M=4$, and $K=[5,7]$ in the Section IV.B scenario. One can see that (11) is satisfied for $K=7$ and violated for $K=5$. The total number of states for such network without occupation control is $I=F^{N M}=4096$. The non-convergence probabilities calculated according to (18) are presented in Table 2 and the equilibrium and transient results for the convergent trials are shown in Fig. 7 for 100 channel realizations. One can see that for $K=7$, the GN algorithm demonstrates the desirable behavior with

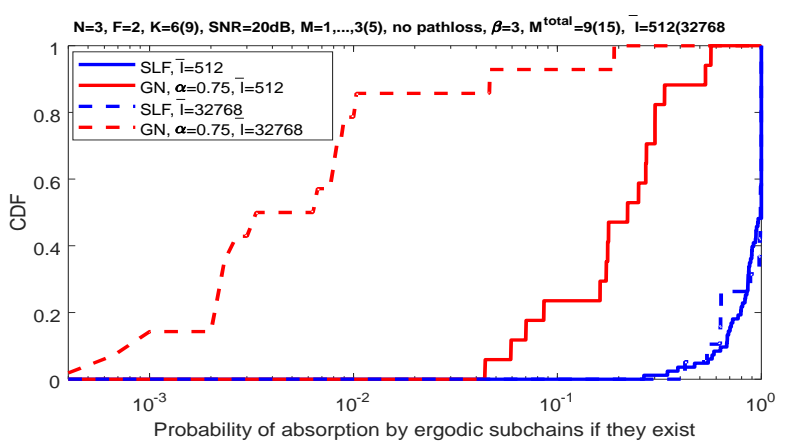

Fig. 6. Probability of absorption by ESs if they exist in scenarios in Fig. 5

convergence probability, rate and equilibrium performance trade-off controllable by $\alpha$. For $K=5$, GN actually collapses to the selfish case, and the performance degrades accordingly as expected. The globally optimal and random allocation results are also shown in Fig. 7a for comparison.

Table 2. DSA with and without occupation control (OC)

\begin{tabular}{|c|c|c|c|c|c|c|}
\hline \multirow{2}{*}{ Algorithm } & \multicolumn{3}{|c|}{ Non-convergence probability, \% } \\
\cline { 2 - 7 } & \multicolumn{3}{|c|}{13.5} & \multicolumn{3}{c|}{$K=7$} \\
\hline SLF, no OC & \multicolumn{3}{|c|}{12.5} & \multicolumn{3}{c|}{15.6} \\
\hline SLF, OC & 1 & 0.75 & 0.5 & 1 & 0.75 & 0.5 \\
\hline$\alpha$ & 13.5 & 13.5 & 13.5 & 0.17 & 0.04 & 0 \\
\hline GN, no OC & 13.5 & 0.03 & 0 & 0.19 & 0.05 & 0 \\
\hline GN, OC & 0.07 & 1.35 & 0.52 & 1.94 & 1.19 & 0.27 \\
\hline SLF/GN & 2.35 & & \multicolumn{3}{c}{} \\
\hline
\end{tabular}
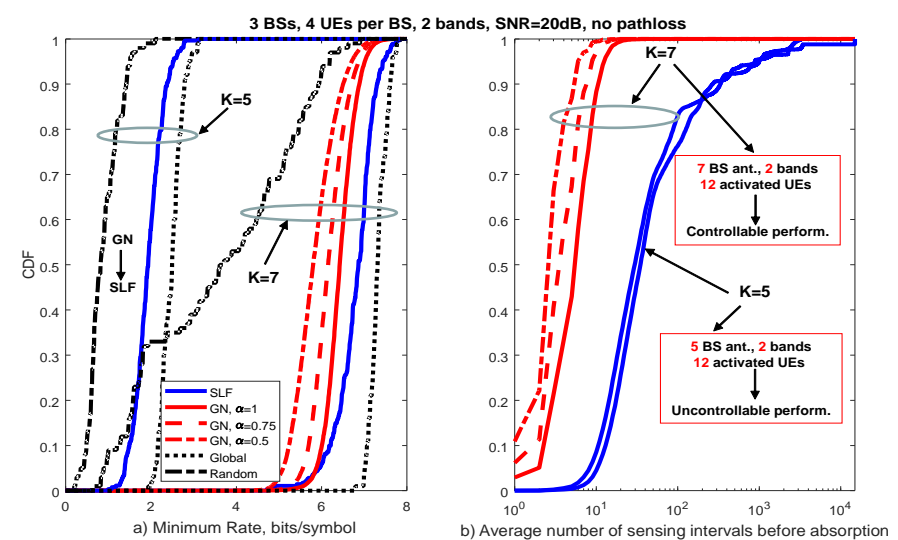

Fig. 7. Convergence results without occupation control

A similar network with occupation control is studied for $\tilde{M}=4$ and $\beta=2$. The corresponding results are shown in Table 2 and Fig. 8. One can see that the desirable controllable results for $K=5$ are back and similar to the $K=7$ performance. The difference is that for $K=7$, all the actual absorbing states contain allocations with all $M^{\text {total }}=N \tilde{M}=F K-\beta=12$ activated UEs, but for $K=5$ only $M^{\text {total }}=F K-\beta=8$ UEs are activated for each absorbing state. Also, it is interesting to note a much faster SLF convergence in Fig. 8b for the case of $K=5$ and $M^{\text {total }}=8$ compared to $K=7$ and $M^{\text {total }}=12$. This is because of much higher average number of SLF ASs in the first scenario as analytically explained in Section IV.C.1 (actually, 12 times higher for the average lower estimate 
(35) and practically zero no AS probability in this case as illustrated in Fig. 2).
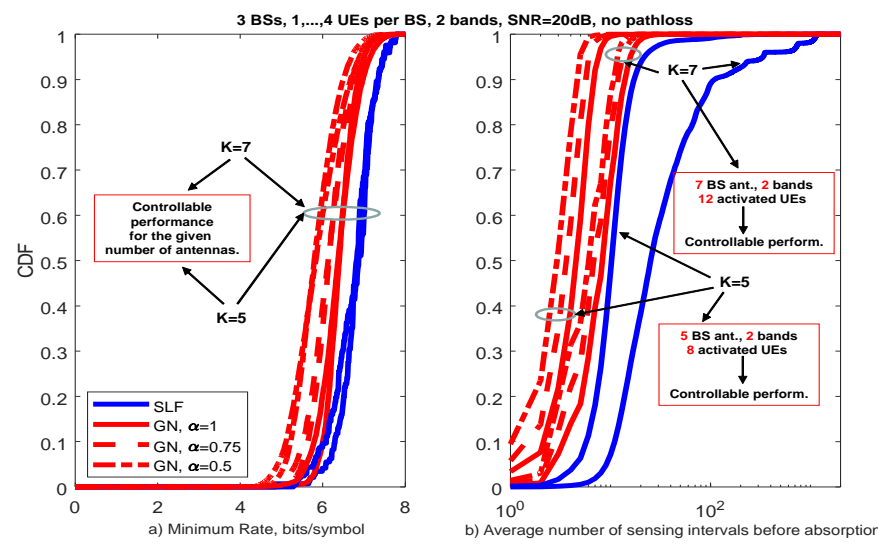

Fig. 8. Convergence results with occupation control

Now, we study effects of a possible partial break of the GN rules in the same scenario for $K=5$ assuming that $\mathrm{BS}_{1}$ follows the SLF algorithm with fixed $M_{1}=\tilde{M}=4$, while $\mathrm{BS}_{2}$ and $\mathrm{BS}_{3}$ run the GN algorithm with the occupation control with $\beta=2$. The total number of Markov chain states is reduced to $I=14400$ in this case because of no occupation control for $\mathrm{BS}_{1}$. The corresponding nonconvergence probabilities are summarized in Table 2. Comparison of the convergence results and the UE number distributions with the corresponding performance for GN with occupation control for all BSs with $K=5$ antennas are given in Figs. 9 and Fig. 10. One can see the following consequences of the selfish behavior of $\mathrm{BS}_{1}: 1$ ). Subsystem 1 always gets the maximum activated UEs and forces other sub-systems to reduce their occupation to maintain the efficient interference mitigation for the available resources as illustrated in Fig. 10. 2). The steady-state minimum data rate performance in Fig. 9a is negligibly increased because of slightly higher selfish equilibrium results for subsystem 1 if and when it converges. 3). The corresponding non-convergence probabilities in Table 2 are significantly higher for the mixed network case compared to the all GN results. 4). The convergence rate results are decreased for the mixed network as one can see in Fig. 9b.

The main observation from the GN rule break effects is that the transient performance degradation in terms of the increased non-convergence probabilities and reduced convergence rate belongs to the whole network including the selfish subsystem, which can be considered as a demotivation for the selfish behavior in the considered rule regulated networks.

\section{Higher-Dimension Decentralized DSA with OcCupation AND Power Control}

An exhaustive local search over all $F^{M_{n}}$ options in (7)(10) may not be feasible for higher dimension networks. One possible simplification can be based on a partial search over some subsets of users $\tilde{\mathcal{M}}_{n}$ and bands $\tilde{\mathcal{F}}_{n}$ with the restricted number of elements $M_{\max } \leq M_{n}$ and $F_{\max } \leq F$

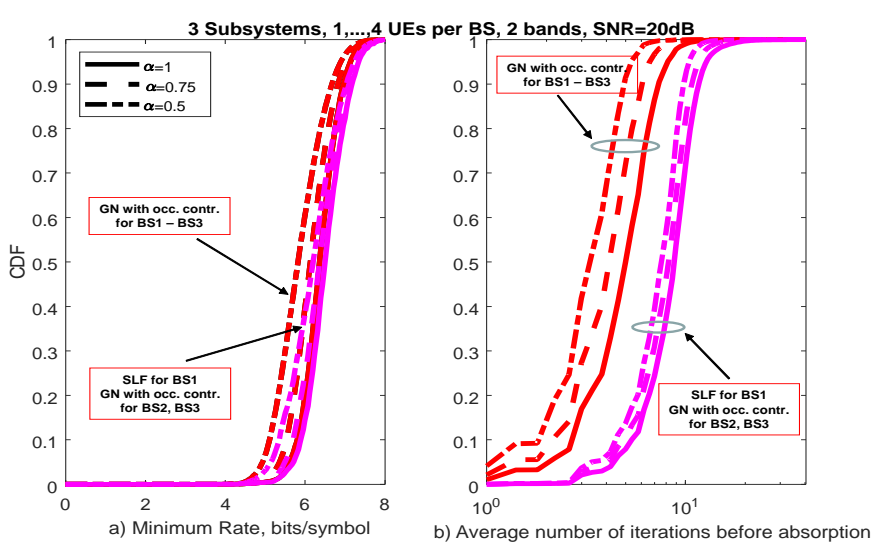

Fig. 9. Convergence results for partial break of the GN rules
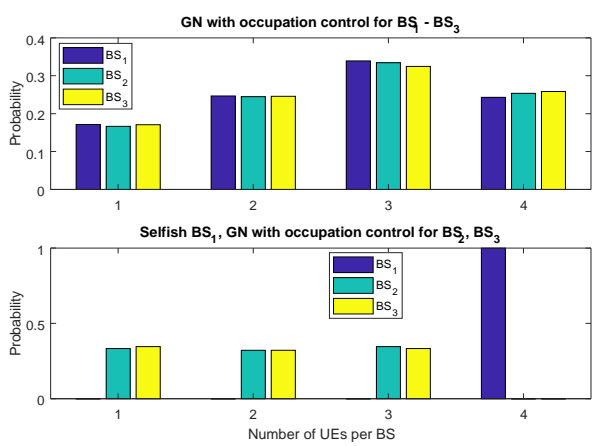

Fig. 10. Distribution of the number of activated UEs with and without partial break of the GN rules

instead of the whole sets of bands and activated UEs in (7)-(10).

One way to define $\tilde{\mathcal{M}}_{n}$ and $\tilde{\mathcal{F}}_{n}$ is to collect the UEs with the lowest SINR for $\tilde{\mathcal{M}}_{n}$ and the fixed number of $F_{\max }$ bands with the highest dimensions of the noise subspace $U_{n f_{j}}, j=1, \ldots, F_{\max }$ for $\tilde{\mathcal{F}}_{n}$ taking into account that $U_{n f}$ needs to be estimated at the sensing interval for the occupation control in any case.

It is well known that power control can significantly improve efficiency of MIMO networks [36]. Generally, in the considered interference limited spectrum sharing case, power adjustments at some subsystems change interference scenarios for other subsystems, which may complicate an efficient power control. It is important to note that this is actually not the case in the strong interference limited scenario if the number of receive antennas at BSs is high enough for rejection of all interference sources. This is clearly follows from equation (22), which shows that SINR practically does not depend on $\mu_{i n t} \gg 1$ in this case. The main reason for the introduced occupation control is to make sure that for the given number of antennas and available bands, the efficient interference mitigation can be maintained. This means that a local power control should not practically affect other subsystems in the considered network environment.

Similarly to [36], one possibility could be maximization of the minimum SINR to improve the weakest local link for 
the given power constraint:

$$
q_{n m}^{2}=Q_{n} \frac{\frac{1}{\mathbf{h}_{d_{n m m n n}}^{*} \mathbf{R}_{d_{n m n}}^{-1} \mathbf{h}_{d_{n m} m n n}}}{\sum_{q=1}^{M_{n}} \frac{1}{\mathbf{h}_{d_{n q} q n n}^{*} \mathbf{R}_{d_{n q n}}^{-1} \mathbf{h}_{d_{n q} q n n}}}, m=1, \ldots, M_{n},
$$

where $Q_{n}=\sum_{m=1}^{M_{n}} q_{n m}^{2}$ is the total power constraint specified in Section II. All the considered algorithms can be implemented with such power control by means of replacing $\mathbf{h}_{d_{n m} m n n}$ with $q_{n m} \mathbf{h}_{d_{n m} m n n}$.

\section{A. Simulation Results: No Pathloss Modeling}

Efficiency of the occupation and power control is illustrated in the first simulation for unlimited number of requested UEs $\tilde{M}_{n}$ for $N=5, F=7, K=15, \alpha=0.5, \beta=3$, $\delta=2, \Delta=1, M_{\max }=4$, and $F_{\max }=3$ with initially 10 activated UEs per sub-system. Taking into account that all sub-systems can sense all activated UEs in the pathloss scenario, the total number of activated UEs for the unlimited $\tilde{M}_{n}$ should be $K F-\beta=102$ in the considered scenario. The simulation results in 100 independent random channels and sensing interval trials are summarized in Figs. 11 and 12. Fig. 11 presents distributions of the steady-stated number of the activated UEs per subsystem after convergence. Fig. 12 shows CDFs of the minimum data rate and convergence rate ${ }^{2}$ for GN with and without power control. It is found that the total number of the activated UEs is 102 in all trials as expected, but different subsystems experience different steady-state number of the activated UEs, mainly depending of particular sensing interval realizations with approximately 20 UEs per subsystem on average as shown in Fig. 11. Fig. 12 illustrates that the power control significantly improves the equilibrium data rate results.

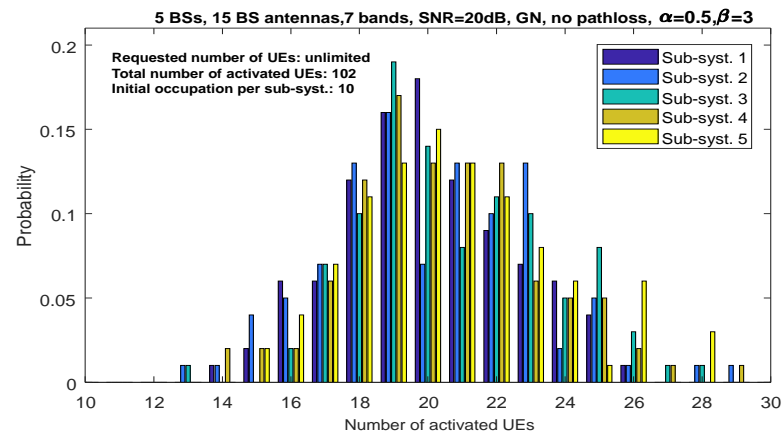

Fig. 11. Distribution of the number of activated UEs per subsystem

\section{B. Simulation Results: Pathloss Modeling}

The main feature of spectrum sharing scenarios with pathloss and shadowing modeling is that different BSs may sense different numbers of activated UEs depending on the pathoss model, BSs and UEs locations and noise space detection parameter $\delta$. Then, the constant total number of

\footnotetext{
${ }^{2}$ The convergence is detected if the occupation control and band allocations do not change for all subsystems in 50 consecutive sensing intervals.
}
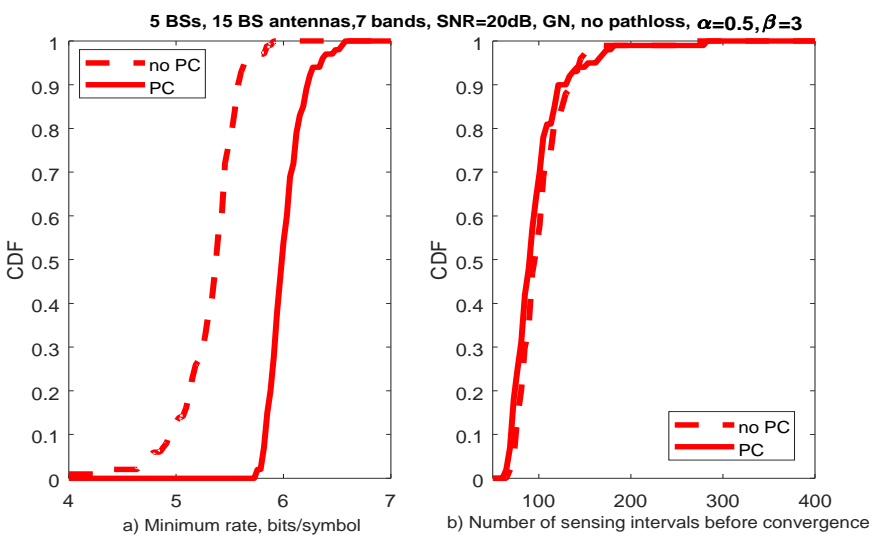

Fig. 12. Equilibrium and transient performance with and without power control

activated UEs for all subsystems in different scenario realizations, as in the no pathloss environment, may not be the case.

Let us consider the same network and algorithm parameters as in Figs. 11 and 12 with a simple pathloss model and parameters similar to [37] assuming a reference distance of $20 \mathrm{~m}$ for $\mathrm{SNR}=20 \mathrm{~dB}$ with the same pathloss for all bands. All BSs and UEs are placed in the $(50 \times 50) \mathrm{m}$ area with the minimum distance between different BSs of $10 \mathrm{~m}, 15 \mathrm{~m}$ radius of sub-system UEs deployment for the corresponding BS locations, and $2 \mathrm{~m}$ minimum distance between any UE and BS. One realization of this scenario is shown in Fig. 13. One can expect that $\mathrm{BS}_{4}$ and $\mathrm{BS}_{5}$ located in center of the considered area may "see" more UEs compared to the $\mathrm{BS}_{1}-\mathrm{BS}_{3}$ located closer to the borders of the area leading to some congestion problems for the corresponding subsystems. To study the location effects on the spectrum sharing performance, we simulate 100 random Rayleigh fading channel realizations and UE locations for the fixed sensing sequence and fixed BS locations as in Fig. 13 for GN with the occupation and power control. The requested number of UEs varies in time as indicated in Fig. 14 by the dashed lines and arrows. Fig. 14a presents the occupation control results for all 5 subsystems in all 100 trials. One particular example is illustrated in Fig. 14b for the occupation control and in Fig. 14c for the target threshold and minimum data rate results for each subsystem. The presented simulation results demonstrate the following: 1). At the first simulation period of 200 sensing intervals, the network is underloaded and all subsystems get their requested 22 activated UEs. 2). At the second and third simulation periods the network becomes overloaded and different subsystems get different number of activated UEs depending on the network geometry leading to variable total network occupation. 3). The simplest occupation situation is observed for isolated subsystems 2 and 3 located close to the area borders with the modest requested number of UEs, particularly, subsystem 2 gets the requested increase of 8 UEs after the first simulation period in all trials and subsystem 3 is not affected by occupation exchange in the central congested area in almost all trials. 4). On the contrary, closely 
located subsystems 1, 4, and 5 significantly affect occupation of each other. 5). In some trials, the significantly, by $18 \mathrm{UEs}$, increased requested occupation of subsystem 1 forces centrally located subsystems 4 and 5 to reduce their occupation as explicitly illustrated in the example in Fig. 14b. 6). Fig. 14c illustrates adaptation of the target thresholds according to (12), (16) following the changes in the signal/interference scenario leading to the fast and reliable DSA convergence. Particularly, the highest targets are observed in the first simulation period because all subsystems are underloaded and spare degrees of freedom are used for diversity gains. At the second simulation interval, the overloaded subsystems 1,4 , and 5 reduce the target thresholds because all the available degrees of freedom up to the occupation control parameter $\beta$ in (15) are used for interference mitigation. After convergence, the minimum data rates stay slightly higher that the target thresholds for all subsystems at all simulation intervals as expected. and reliable DSA convergence.

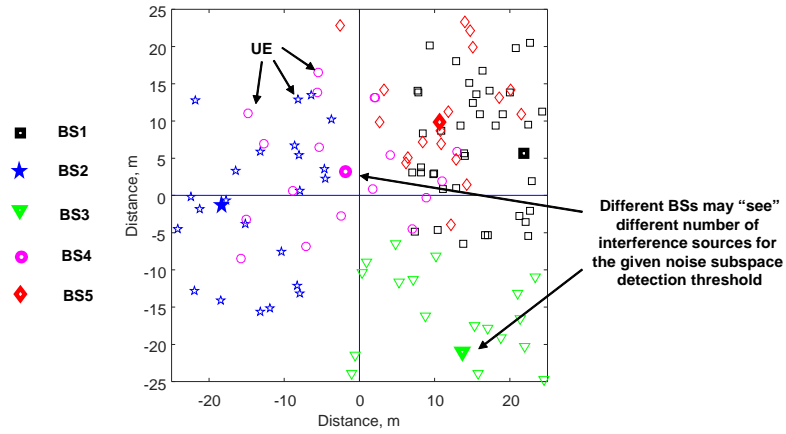

Fig. 13. Network geometry
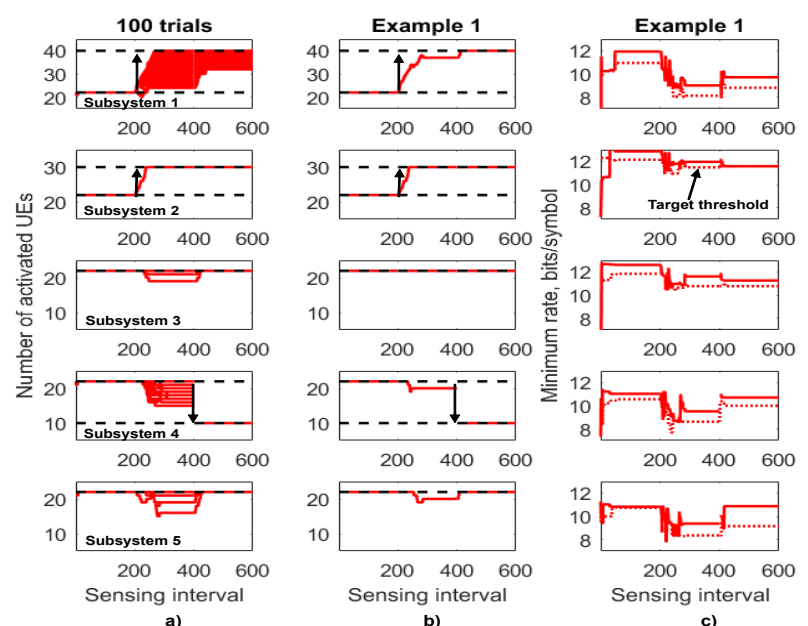

Fig. 14. Occupation control and minimum data rate results for network geometry in Fig. 13

The situation, where some spectrum sharing nodes may force other network players to relocate some of their UEs to other groups of bands in the case of limited resources actually raises a spectrum occupation fairness question in the proposed spectrum sharing approach. There are many

ways to address this problem, for example, different allowed number of antennas for congested and non-congested areas, asymmetric conditions for increasing/decreasing occupation control, etc. This problem deserves further investigation.

It is worth emphasizing that GN convergence is registered for all presented trials and SLF non-convergence is found in wast majority of trials for higher dimension networks (the SLF results are not shown because of that). This illustrates our expectation in Section IV.D regarding a possibility of a very low probability of GN non-convergence for higher dimension networks even if short ergodic subchain may exist for some channel realizations.

\section{Simulation Results: Time varying channels}

To illustrate channel variation effects on the considered algorithms we assume the same network geometry as in Fig. 13 for $N=[2,4], F=5, K=10, \tilde{M}=15, \alpha=0.1, \beta=5$, $\delta=2, \Delta=1, M_{\max }=4, F_{\max }=3, \sigma^{2}=2.5 \cdot 10^{-10}$ and use the IEEE $802.11 \mathrm{n}$ indoor narrow band channel model "A" [26] for all links and bands in the network depending on the distance for $2.4 \mathrm{GHz}$ central frequency, one wavelength antenna spacing and $1.2 \mathrm{~km} / \mathrm{h}$ Doppler speed specified in [26] for indoor scenarios. A lower value of the control parameter $\alpha$ is used in this simulation compared to the stationary channel scenarios to allow a faster tracking of time varying channels. We simulate GN with occupation control together with SLF and random allocation for the fixed initial number of activated UEs assuming that one randomly selected BS is sensing every $10 \mathrm{~ms}$. The minimum data rate performance is plotted in Fig. 15a. Fig. $15 \mathrm{~b}$ indicates the time instances where the GN band allocation updates are actually performed (the SLF updates are performed at every sensing interval). Eventually, Fig. 15c shows the number of GN activated UEs per subsystem and totally in the network. Initially, 2 subsystems with $15 \mathrm{UEs}$ each are activated and then 2 other subsystems with all their UEs are also activated for the rest of the simulation as shown in Fig. 15c. The following observations can be made from the presented simulation results: 1). Initially, the available number of degrees of freedom allow efficient interference mitigation for all activated UEs in the network leading to the similar performance for all algorithms including the random allocation. The occupation control and band allocation updates are not actually needed. 2). After activation of 2 more subsystems, the occupation control and relatively frequent band allocation updates allow the GN algorithm to significantly outperform two other solutions as one can see in Fig. 15a. 3). The occupation control reacts to the changes in the interference environment rather than to Doppler channel variations. This situation suggests that activation of the occupation control may be less frequent compared to the band allocations.

\section{CONCLUSIONS}

A solution for decentralized spectrum sharing with reliable performance targets has been proposed, which is based on the extension of the rule regulated GN DSA by means 

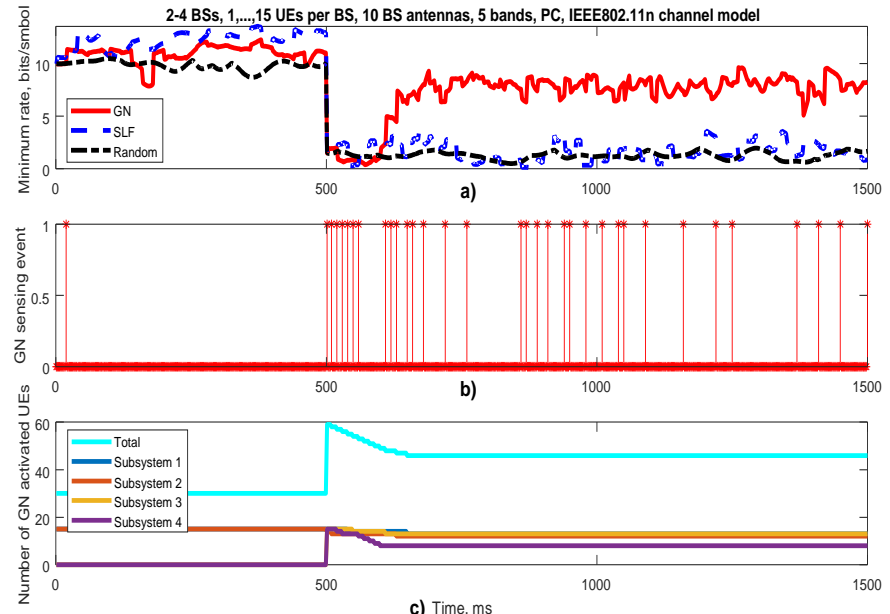

Fig. 15. DSA algorithm comparison for IEEE 802.11n channel model

of the adaptive spectrum occupation control. Analytical estimates for absorbing state statistics have been derived that allow prediction of convergence properties and applicability areas of different DSA algorithms. An absorbing Markov chain DSA model has been extended to the occupation control case with adaptive performance targets. Performance of decentralized rule regulated GN DSA algorithms have been studied by means of semi-analytical investigation and simulations to show a possibility of spectrum sharing with the desired and controllable equilibrium and transient behavior without any explicit communication and cooperation between spectrum sharing subsystems. Spectrum occupation fairness, adaptive ranking of users selected for activation, and finite amount of data estimation effects could be subjects for further investigation.

\section{ACKNOWLEDGMENT}

We would like to acknowledge the support of the University of Surrey 5GIC (www.surrey.ac.uk/5gic) members for this work. The authors are thankful for Dr. Y. I. Abramovich for fruitful discussions.

\section{REFERENCES}

[1] J. G. Andrews, S. Buzzi, W. Choi, S. V. Hanly, A. Lozano, A. C. K. Soong, J. C. Zhang, "What will 5G be?" IEEE J. Sel. Areas Commun., vol. 32, no. 6, pp. 1065-1082, 2014.

[2] "The future role of spectrum sharing for mobile and wireless data services: Licensed sharing, Wi-Fi, and dynamic spectrum access," Ofcom, Statement Report, Apr. 2014.

[3] "Spectrum sharing in wireless networks: Fairness, efficiency, and security," Eds. J. D. Matyjas, S. Kumar, F. Hu, CRS Press, 2016.

[4] N. Zhang, S. Zhang, J. Zheng, X. Fang, J. W. Mark, X. Shen, "QoE driven decentralized spectrum sharing in 5G networks: Potential game approach," IEEE Trans. Vehic. Technol., vol. 66, no. 9, pp. 7797-7808, Sept 2017

[5] A. K. Bairagi, S. F. Abedin, N.H. Tran, D. Niyato, C. S. Hong, "QoE-enabled unlicensed spectrum sharing in 5G: A gametheoretic approach," IEEE Access, vol. 6, pp. 50538-50554, Sept. 2018.

[6] M. Kamel, W. Hamouda, A. Youssef, "Ultra-dense networks: A survey," IEEE Commun. Surveys and Tutorials, vol. 18, no. 4, pp. 2522-2542, May 2016.

[7] $5 \mathrm{G}$ White Paper, Next Generation Mobile Networks (NGMN) Alliance, 17 Feb. 2015. Available: https://www.ngmn.org/uploads/media/NGMN_5G_White_Paper _V1_0.pdf
[8] L. Cao, H. Zheng, "Distributed rule-regulated spectrum sharing," IEEE J. Sel. Areas Commun., vol. 26, no. 1, pp. 130-145, Jan. 2008.

[9] L. Sanabria-Russo, J. Barcelo, B. Bellalta, F. Gringoli, "A high efficiency MAC protocol for WLANs: Providing fairness in dense scenarios," IEEE Trans. Networking, vol. 25, no. 1, pp. 492-505, Feb. 2017.

[10] J. Herzen, A. Banchs, V. Shneer, P. Thiran, "CSMA/CA in time and frequency domains," in Proc. ICNP, Nov.2015.

[11] B. Mawlawi, J.-B. Dore, N. Lebedev, J.-M. Gorce, "Multiband CSMA/CA with RTS-CTS strategy," in Proc. WiMob, Oct. 2014

[12] O. Ashagi, S. Murphy, L. Murphy, "A distributed approach to interference mitigation between OFDM-based 802.16 systems operating in license-exempt spectrum," in Proc. ICC, pp. 4855-4860, Jun. 2007.

[13] G. Arslan, M. F. Demirkol, Y. Song, "Equilibrium efficiency improvement in MIMO interference systems: A decentralized stream control approach," IEEE Trans. Wireless Commun., vol. 6, no. 8, pp. 2984-2993, Aug. 2007.

[14] G. Scutari, D. Palomar, S. Barbarossa "Cognitive MIMO radio," IEEE Signal Proc. Mag., pp. 46-59, Nov. 2008.

[15] G. Scutari, D. Palomar, S. Barbarossa, "Competitive design of multiuser MIMO systems based on game theory: A unified view," IEEE J. Sel. Areas Commun., vol. 26, no. 7, pp. 1089-1103, Sep. 2008.

[16] G. Scutari, D. Palomar, "MIMO cognitive radio: A game theoretic approach," IEEE Trans. Sig. Proc., vol. 58, no. 2, pp. 761779 , Feb. 2010

[17] G. Scutari, D. Palomar, S. Barbarossa, "The MIMO iterative waterfilling algorithm," IEEE Trans. Sig. Proc., vol. 57, no. 5, pp. 1917-1935, May 2009.

18] S. M. Perlaza, S. Lasaulce. M. Debbah, "Equilibria of channel selection games in parallel multiple access channels," EURASIP J. Wireless Commun. and Networking, pp. 1-23, Dec. 2013.

[19] M. Y. ElNainay, A. B. MacKenzie, "Efffect of non-cooperation on dynamic spectrum cognitive networks," in Proc. IWCMC, June 2009

[20] R. Menon, A. B. MacKenzie, R. M. Buehrer, J. H. Reed, "A game theoretic framework for interference avoidance in ad hoc networks, in Proc. GLOBECOM, Nov. 2007.

[21] R. Southwell, J. Huang, B. Shou, "Physical interference model based spectrum sharing with generalized spatial congestion games," in Proc. ICCS, pp. 260-264, 2012

22] A. M. Kuzminskiy, Y. I. Abramovich, "Decentralized dynamic spectrum allocation based on adaptive antenna array interference mitigation diversity," IEEE Trans. on Sig. Proc., vol. 58, no.4, pp. 2246-2260, Apr. 2010

[23] A. M. Kuzminskiy, P. Xiao, R. Tafazolli, "Good neighbor distributed beam scheduling in coexisting multi-RAT networks, in Proc. WCNCW, pp. 226-260, Apr. 2018.

[24] A. M. Kuzminskiy, Y. I. Abramovich, P. Xiao, R. Tafazolli, "Spectrum sharing efficiency analysis in rule regulated networks with decentralized occupation control," in Proc. PIMRC, Sept. 2016.

[25] M. Renfors, F. Bader, L. Baltar, D. Le Ruyet, D. Roviras, P. Mege, M. Haardt, T. H. Stitz, "On the use of filter bank based multicarrier modulation for professional mobile radio," in Proc. VTC Spring, 2013

26] L. Schumacher, B. Dijkstra, "Description of a MATLAB implementation of the indoor MIMO WLAN channel model proposed by the IEEE 802.11 TGn channel model special committee," Jan. 2004. Available: https://staff.info.unamur.be/lsc/Publications/2004/ implementation_note_TGn_v31.pdf

[27] A. M. Kuzminskiy, Y. I. Abramovich, "Decentralized "good neighbor" DSA based on adaptive antenna array interference mitigation diversity: Finite amount of data effects, in Proc. CrownCom, June 2010

[28] B. L. Fox, D. M. Landi, "An algorithm for identifying the ergodic subchains and transient states of a stochastic matrix," Communications of the ACM, vol. 11, no. 9, pp. 619-621, Sept. 1968.

[29] J. G. Kemeny, G. L. Thompson, "Introduction to finite mathematics," Prentice-Hall, N. J., 1966.

[30] D. A. Schmidt, C. Shi, R. À. Berry, M. L. Honig, W. Utschick, "Comparison of distributed beamforming algorithms for MIMO interference networks," IEEE Trans. on Sig. Proc., vol. 61, no. 13, pp. 3476-3489, July 2013.

[31] G. H. Golub, C. F. Van Loan, "Matrix Computations," Johns Hopkins Univ. Press, Baltimore, 1996

[32] G. J. Foschini, M. J. Gans, "On limits of wireless communications in fading environment when using multiple antennas," Wireless Pers. Commun. vol. 6, no. 3, pp. 311-335, Mar. 1998.

[33] L. S. Gradshteyn, Î. M. Ryzhik, "Tables of integrals, series, and 
products," Academic, New York, 1994

[34] G. Jaklic, V. Vitrih, E. Zagar, "Closed form formula for the number of restricted compositions," Bull. Australian Math. Soc., vol. 81, pp. 289-297, 2010.

[35] "Handbook of Mathematical Functions with Formulas, Graphs, and Mathematical Tables." Eds. M. Abramowitz, I. A. Stegun. Washington, D.C., 1964

[36] A. Pascual-Iserte, A. I. Perez-Neira, M. A. Lagunas, "On power allocation strategies for maximum signal to noise and interference ratioin in an OFDM-MIMO system," IEEE Trans. Wireless Commun., vol. 3, no. 3, pp. 808-820, May 2004.

[37] M. Trivellato, F. Boccardi, H. Huang, "Zero-forcing vs unitary beamforming in multiuser MIMO systems with limited feedback," in Proc. PIMRC, Sept. 2008. 